\title{
2-Aminoquinazolines by Chan-Evans-Lam Coupling of Guanidines with (2-Formylphenyl)boronic Acids
}

\author{
Vitalii V. Solomin ${ }^{\mathrm{a}, \mathrm{b}}$ \\ Alberts Seins ${ }^{\mathrm{a}, \mathrm{b}}$ \\ Aigars Jirgensons*a,b (D) \\ a Latvian Institute of Organic Synthesis, Aizkraukles 21, Riga, \\ LV-1006, Latvia \\ aigars@osi.Iv \\ b Faculty of Materials Science and Applied Chemistry, Riga \\ Technical University, P. Valdena Str. 3, Riga LV-1048, Latvia
}
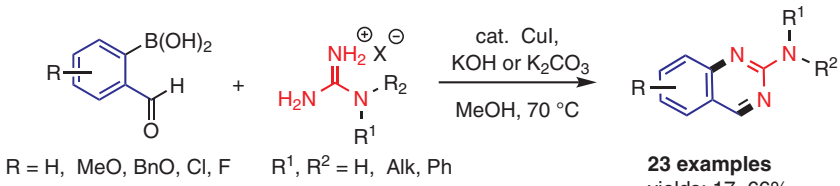

yields: $17-66 \%$

\author{
Received: 30.04.2020 \\ Accepted after revision: 10.06 .2020 \\ Published online: 08.07 .2020 \\ DOI: 10.1055/s-0040-1707080; Art ID: st-2020-k0256-I \\ License terms: (C) \\ (c) 2020. The Author(s). This is an open access article published by Thieme under the \\ terms of the Creative Commons Attribution License, permitting unrestricted use, dis- \\ tribution and reproduction, so long as the original work is properly cited. \\ (https://creativecommons.org/licenses/by/4.0/)
}

Abstract A new method is presented for the synthesis of 2-aminoquinazolines, which is based on a Chan-Evans-Lam coupling of (2formylphenyl)boronic acids with guanidines. Relatively mild conditions involving the use of inexpensive Cul as a catalyst and methanol as a solvent permit the application of the method to a wide range of substrates. Nonsubstituted, N-monosubstituted, and N,N-disubstituted guanidines can be used as reactants to give the corresponding 2-aminoquinazolines in moderate yields from readily available (2-formylphenyl)boronic acids.

Key words guanidines, Chan-Evans-Lam coupling, quinazolines, copper catalysis, formylphenylboronic acids

2-Aminoquinazoline is an important substructure for the development of pharmaceutically relevant compounds, especially for the discovery of kinase inhibitors. ${ }^{1-6}$ A number of methods for the construction of 2-aminoquinazolines are know; ${ }^{7-16}$ However, there are only a few approaches that exploit guanidines as reaction components. orthoHalobenzaldehydes and aryl ketones can be condensed with guanidines in most cases if they contain an additional electron-withdrawing group that facilitates an $\mathrm{S}_{\mathrm{N}} \mathrm{Ar}$ reaction. ${ }^{3,6,13,14,17,18}$ For nonactivated substrates, a copper-catalyzed arylation of guanidines with aryl bromides has been described as a useful method for accessing 2-aminoquinazolines. ${ }^{19}$ However, the reaction conditions are very harsh (DMF, $\left.120^{\circ} \mathrm{C}\right)$, which limits the scope of this approach.
The Chan-Evans-Lam coupling ${ }^{20-24}$ is an attractive C-N bond-forming reaction that proceeds under relatively mild copper-catalyzed conditions and tolerates alcoholic solvents. To our knowledge, the only precedent for accessing quinazoline derivatives by using Chan-Evans-Lam coupling is a synthesis of quinazolonimines by arylation of $\mathrm{N}, \mathrm{N}-$ disubstituted guanidines, formed in situ, with (2-cyanophenyl)boronic acids. ${ }^{25}$ To facilitate our kinase-inhibitordevelopment program, we examined whether the ChanEvans-Lam coupling might also be applicable to the synthesis of aminoquinazolines under mild conditions by using readily available reagents.

A screening of the reaction conditions was performed for the synthesis of unsubstituted quinazoline-2-amine (3). Representative results are reported in Table 1 [see Supporting Information (SI) for the full set of experiments]. Due to the polarity of the product 3 , its purification by chromatography was difficult, and it was therefore purified by trituration from ethyl acetate. An identical scale and workup were applied in all experiments to permit comparison of the effects of other reaction parameters. Methanol as a reaction solvent, $\mathrm{CuI}$ as a catalyst, and $\mathrm{K}_{2} \mathrm{CO}_{3}$ as a base were found to be productive conditions for the formation of quinazoline2-amine (3) from (2-formylphenyl)boronic acid (1) and guanidine hydrochloride (2a) (Table 1, entries 1 and 2).

Excess amounts of the base and guanidine were beneficial in improving the yield of product 3 (Table 1, entry 2 ). Other copper catalysts [ $\mathrm{CuCl}$ and $\mathrm{Cu}(\mathrm{OAc})_{2}$ ] were found to be less efficient than $\mathrm{CuI}$ (entries 3 and 4). The use of $\mathrm{KOH}$ as base improved the yield of product $\mathbf{3}$ when an excess of guanidine was used (entries 5 and 6). EtOH could also be successfully used as the reaction solvent (entry 7). Guanidine carbonate ( $\mathbf{2 b}$ ) as a reactant gave a reduced yield of quinazoline 3 (entries 8 and 9). All the experiments listed in Table 1 were performed open to air to ensure reoxidation 
Table 1 Chan-Evans-Lam Conditions for the Synthesis of 2-Aminoquinazoline (3)

\begin{tabular}{|c|c|c|c|c|c|c|}
\hline Entry & Solvent & $\operatorname{Temp}\left({ }^{\circ} \mathrm{C}\right)$ & Reactant (equiv) & Catalyst & Base (equiv) & Yield ${ }^{b}(\%)$ \\
\hline 1 & $\mathrm{MeOH}$ & 70 & $2 a(1.5)$ & Cul & $\mathrm{K}_{2} \mathrm{CO}_{3}(2.5)$ & 31 \\
\hline 2 & $\mathrm{MeOH}$ & 70 & $2 a(2.5)$ & Cul & $\mathrm{K}_{2} \mathrm{CO}_{3}(3)$ & 44 \\
\hline 3 & $\mathrm{MeOH}$ & 70 & $2 \mathbf{a}(2.5)$ & $\mathrm{Cu}(\mathrm{OAc})_{2}$ & $\mathrm{~K}_{2} \mathrm{CO}_{3}(3)$ & 35 \\
\hline 4 & $\mathrm{MeOH}$ & 70 & $2 a(2.5)$ & $\mathrm{CuCl}$ & $\mathrm{K}_{2} \mathrm{CO}_{3}(3)$ & 23 \\
\hline 5 & $\mathrm{MeOH}$ & 70 & $2 a(1.5)$ & Cul & $\mathrm{KOH}(1.5)$ & 34 \\
\hline 6 & $\mathrm{MeOH}$ & 70 & $2 a(3)$ & Cul & $\mathrm{K}_{2} \mathrm{CO}_{3}(3)$ & $51(65)^{c}$ \\
\hline 7 & EtOH & 90 & $2 \mathbf{a}(3)$ & Cul & $\mathrm{K}_{2} \mathrm{CO}_{3}(3)$ & 52 \\
\hline 8 & $\mathrm{MeOH}$ & 70 & $\mathbf{2 b}(3)$ & Cul & - & 13 \\
\hline 9 & $\mathrm{MeOH}$ & 70 & $\mathbf{2 b}(1.5)$ & Cul & $\mathrm{KOH}(3)$ & 17 \\
\hline
\end{tabular}

a Reactions were performed open to the air, reaction time: 12-17 h.

b Purified by trituration with EtOAc to a purity of $>98 \%$.

C NMR yield with 1,3,5-trimethoxybenzene as an internal standard.

of the copper catalyst. Performing the reaction under an oxygen atmosphere or adding hydrogen peroxide did not substantially improve the yield of product $\mathbf{3}$ (see SI).

Next, (2-formylphenyl)boronic acid (1) was treated with a range of guanidines under the most productive reaction conditions (Table 1 , entry 6). ${ }^{26}$ Both $\mathrm{N}$-monosubstituted guanidines $\mathbf{4 a}-\mathbf{g}$ and $\mathrm{N}, \mathrm{N}-$ disubstituted guanidines $(\mathbf{4 h}-\mathbf{j})$ provided the corresponding 2 -aminoquinazolines $\mathbf{5 a}-\mathbf{j}$ in fairly good yields (Table 2 ).

Several (2-formylphenyl)boronic acids 6a-f were next explored as substrates for the synthesis of aminoquinazolines 7a-f and 8a-f (Table 3). Both guanidines $2 \mathbf{a}$ and $\mathbf{4 a}$ gave the expected products but the isolated yields were generally somewhat higher in the case of the $N$-methylsubstituted guanidine 4a (Table 3; entries 3 and 4, 5 and 6, 7 and 8 ).

Boronic acid derivatives such as the pinacolate ester 9a and the trifluoroborate $\mathbf{9 b}$ were also competent substrates, providing aminoquinazoline derivative $\mathbf{5 a}$ in yields comparable to those from boronic acid $\mathbf{1}$ (Scheme 1 ). These results complement the relatively few reported cases of the use of boronic acid derivatives as partners for Chan-Evans-Lam coupling. ${ }^{27,28}$

In contrast, the boronic acids $10 a$ and $10 b$ bearing a keto group were found to be unsuitable reaction partners for the synthesis of the corresponding quinazolines 11a and 11b (Scheme 2). In the case of these substrates, complex mixtures were obtained containing the O-arylation products $\mathbf{1 2 a}$ and $\mathbf{1 2 b}$ as the only identifiable byproducts. The failure of (2-acylphenyl)boronic acids 10a and $\mathbf{1 0 b}$ to give the expected products implies that the formation of an arylidene guanidine is the first step in the synthesis of aminoquinazolines $\mathbf{3}, \mathbf{5}, \mathbf{7}$, and $\mathbf{8}$, followed by intramolecular arylation

Table 2 Guanidine Scope for the Synthesis of Aminoquinazolines

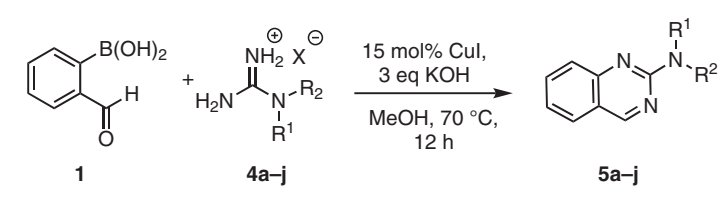

\begin{tabular}{|c|c|c|c|c|c|}
\hline Entry & Guanidine & $\mathrm{R}^{1}$ & $\mathrm{R}^{2}$ & Product & Yield $(\%)$ \\
\hline 1 & $4 a^{b}$ & $\mathrm{H}$ & Me & $5 a$ & 63 \\
\hline 2 & $4 b^{c}$ & $\mathrm{H}$ & $\mathrm{Ph}$ & $5 b$ & 56 \\
\hline 3 & $4 c^{\mathrm{b}}$ & $\mathrm{H}$ & $\mathrm{Bn}$ & $5 c$ & $66^{d}$ \\
\hline 4 & $4 d^{b}$ & $\mathrm{H}$ & $\mathrm{Ph}\left(\mathrm{CH}_{2}\right)_{2}$ & $5 d$ & 52 \\
\hline 5 & $4 e^{b}$ & $\mathrm{H}$ & $\mathrm{Me}\left(\mathrm{CH}_{2}\right)_{4}$ & $5 e$ & 54 \\
\hline 6 & $4 f^{b}$ & $\mathrm{H}$ & cyclopentyl & $5 f$ & 55 \\
\hline 7 & $\mathbf{4} \mathbf{g}^{\mathrm{b}}$ & $\mathrm{H}$ & Cy & $5 g$ & 37 \\
\hline 8 & $4 h^{e}$ & Me & Me & $5 \mathrm{~h}$ & 43 \\
\hline 9 & $4 i^{\mathrm{b}}$ & \multicolumn{2}{|c|}{$\left(\mathrm{CH}_{2}\right)_{4}$} & $5 i$ & 47 \\
\hline 10 & $4 \mathrm{j}^{\mathrm{b}}$ & \multicolumn{2}{|c|}{$\left(\mathrm{CH}_{2}\right)_{2} \mathrm{O}\left(\mathrm{CH}_{2}\right)_{2}$} & $5 \mathbf{j}$ & 39 \\
\hline
\end{tabular}

a Purified by column chromatography unless stated otherwise.

${ }^{b}$ Hydrochloride salt.

c Carbonate salt.

d Purified by trituration with EtOAc.

e Sulfate salt. 
Table 3 Boronic Acid Scope for the Synthesis of Aminoquinazolines

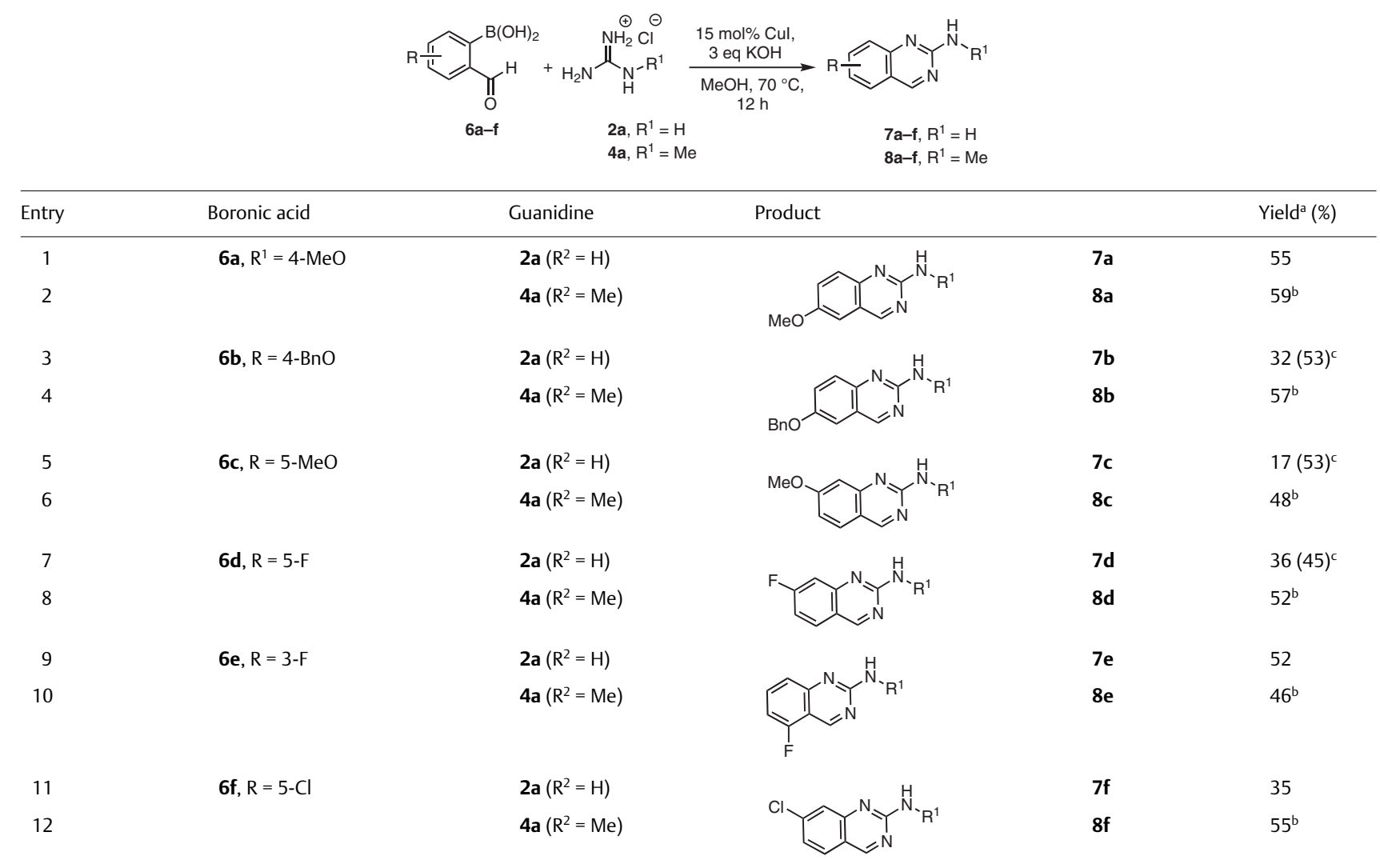

a Purified by trituration with EtOAc unless stated otherwise.

${ }^{b}$ Purified by column chromatography.

' NMR yield with 1,3,5-trimethoxybenzene as an internal standard.

$$
\begin{aligned}
& \text { } \\
& 9 a, X=B P i n \quad 4 a \quad 58 \% \text { from } 9 a \quad 5 a \\
& 9 \mathrm{ab}, \mathrm{X}=\mathrm{BF}_{3} \mathrm{~K} \quad \mathrm{51} \% \text { from } 9 \mathrm{~b}
\end{aligned}
$$

Scheme 1 Synthesis 2-aminoquinazoline from a boronic acid ester and trifluoroborate

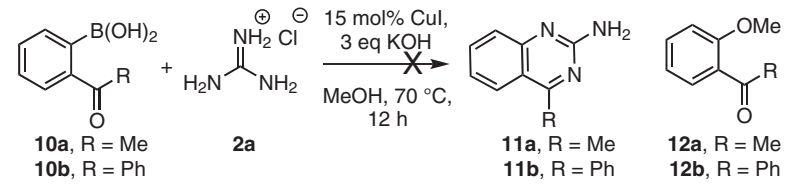

Scheme 2 Attempt to condense keto-group-containing boronic acids with guanidine

In summary, 2-aminoquinazolines can be obtained by Chan-Evans-Lam coupling of (2-formylphenyl)boronic acids with guanidines. The relatively mild reaction conditions permit the use of this method for the synthesis of pharmacologically relevant compounds bearing a 2-aminoquinazoline scaffold.

\section{Funding Information}

H2020 MSC-ITN project CARTNET “Combating Antimicrobial Resistance Training Network”, Grant agreement ID: 765147

\section{Acknowledgment}

We thank Dr. Janis Veliks for his advice and revision of the data.

\section{Supporting Information}

Supporting information for this article is available online at https://doi.org/10.1055/s-0040-1707080.

\section{References and Notes}

(1) Bathini, Y.; Singh, I.; Harvey, P. J.; Keller, P. R.; Singh, R.; Micetich, R. G.; Fry, D. W.; Dobrusin, E. M.; Toogood, P. L. Bioorg. Med. Chem. Lett. 2005, 15, 3881.

(2) Esvan, Y. J.; Zeinyeh, W.; Boibessot, T.; Nauton, L.; Théry, V.; Knapp, S.; Chaikuad, A.; Loaëc, N.; Meijer, L.; Anizon, F.; Giraud, F.; Moreau, P. Eur. J. Med. Chem. 2016, 118, 170. 
(3) Zeinyeh, W.; Esvan, Y. J.; Josselin, B.; Baratte, B.; Bach, S.; Nauton, L.; Théry, V.; Ruchaud, S.; Anizon, F.; Giraud, F.; Moreau, P. Bioorg. Med. Chem. 2019, 27, 2083.

(4) DiMauro, E. F.; Newcomb, J.; Nunes, J. J.; Bemis, J. E.; Boucher, C.; Buchanan, J. L.; Buckner, W. H.; Cee, V. J.; Chai, L.; Deak, H. L.; Epstein, L. F.; Faust, T.; Gallant, P.; Geuns-Meyer, S. D.; Gore, A.; Gu, Y.; Henkle, B.; Hodous, B. L.; Hsieh, F.; Huang, X.; Kim, J. L.; Lee, J. H.; Martin, M. W.; Masse, C. E.; McGowan, D. C.; Metz, D.; Mohn, D.; Morgenstern, K. A.; Oliveira-dos-Santos, A.; Patel, V. F.; Powers, D.; Rose, P. E.; Schneider, S.; Tomlinson, S. A.; Tudor, Y.-Y.; Turci, S. M.; Welcher, A. A.; White, R. D.; Zhao, H.; Zhu, L.; Zhu, X. J. Med. Chem. 2006, 49, 5671.

(5) Vasbinder, M. M.; Aquila, B.; Augustin, M.; Chen, H.; Cheung, T.; Cook, D.; Drew, L.; Fauber, B. P.; Glossop, S.; Grondine, M.; Hennessy, E.; Johannes, J.; Lee, S.; Lyne, P.; Mörtl, M.; Omer, C.; Palakurthi, S.; Pontz, T.; Read, J.; Sha, L.; Shen, M.; Steinbacher, S.; Wang, H.; Wu, A.; Ye, M. J. Med. Chem. 2013, 56, 1996.

(6) Li, C.; Shan, Y.; Sun, Y.; Si, R.; Liang, L.; Pan, X.; Wang, B.; Zhang, J. Eur. J. Med. Chem. 2017, 141, 506.

(7) Li, J.-S.; Fan, Y.-H.; Zhang, Y.; Marky, L. A.; Gold, B. J. Am. Chem. Soc. 2003, 125, 2084.

(8) Bathini, Y.; Sidhu, I.; Singh, R.; Micetich, R. G.; Toogood, P. L. Tetrahedron Lett. 2002, 43, 3295.

(9) Chen, X.; Han, J.; Zhu, Y.; Yuan, C.; Zhang, J.; Zhao, Y. Chem. Commun. 2016, 52, 10241.

(10) Liu, Q.; Zhao, Y.; Fu, H.; Cheng, C. Synlett 2013, 24, 2089.

(11) Sasse, K. Synthesis 1978, 379.

(12) Kikelj, D. In Science of Synthesis, Vol. 16; Yamamoto, Y.; Shinkai, I., Ed.; Thieme: Stuttgart, 2004, Chap. 16.3573.

(13) Babu, D. S.; Srinivasulu, D.; Kotakadi, V. S. Chem. Heterocycl. Compd. 2015, 51, 60.

(14) Smith, A. L.; Andrews, K. L.; Beckmann, H.; Bellon, S. F.; Beltran, P. J.; Booker, S.; Chen, H.; Chung, Y.-A.; D’Angelo, N. D.; Dao, J.; Dellamaggiore, K. R.; Jaeckel, P.; Kendall, R.; Labitzke, K.; Long, A. M.; Materna-Reichelt, S.; Mitchell, P.; Norman, M. H.; Powers, D.; Rose, M.; Shaffer, P. L.; Wu, M. M.; Lipford, J. R. J. Med. Chem. 2015, 58, 1426.

(15) Pandya, A. N.; Villa, E. M.; North, E. J. Tetrahedron Lett. 2017, 58, 1276.

(16) Zhou, G.; Aslanian, R.; Gallo, G.; Khan, T.; Kuang, R.; Purakkattle, B.; Ruiz, M. D.; Stamford, A.; Ting, P.; Wu, H.; Wang, H.; Xiao, D.; Yu, T.; Zhang, Y.; Mullins, D.; Hodgson, R. Bioorg. Med. Chem. Lett. 2016, 26, 1348.

(17) Bollenbach, M.; Salvat, E.; Daubeuf, F.; Wagner, P.; Yalcin, I.; Humo, M.; Letellier, B.; Becker, L. J.; Bihel, F.; Bourguignon, J.-J.; Villa, P.; Obrecht, A.; Frossard, N.; Barrot, M.; Schmitt, M. Eur. J. Med. Chem. 2018, 147, 163.

(18) Huang, K. H.; Barta, T. E.; Rice, J. W.; Smith, E. D.; Ommen, A. J.; Ma, W.; Veal, J. M.; Fadden, R. P.; Barabasz, A. F.; Foley, B. E.; Hughes, P. F.; Hanson, G. J.; Markworth, C. J.; Silinski, M.; Partridge, J. M.; Steed, P. M.; Hall, S. E. Bioorg. Med. Chem. Lett. 2012, 22, 2550.

(19) Huang, X.; Yang, H.; Fu, H.; Qiao, R.; Zhao, Y. Synthesis 2009, 2679.
(20) Chan, D. M. T.; Monaco, K. L.; Wang, R.-P.; Winters, M. P. Tetrahedron Lett. 1998, 39, 2933.

(21) Evans, D. A.; Katz, J. L.; West, T. R. Tetrahedron Lett. 1998, 39, 2937.

(22) Lam, P. Y. S.; Clark, C. G.; Saubern, S.; Adams, J.; Winters, M. P.; Chan, D. M. T.; Combs, A. Tetrahedron Lett. 1998, 39, 2941.

(23) Chen, J.-Q.; Liu, X.; Guo, J.; Dong, Z.-B. Eur. J. Org. Chem. 2020, 2414.

(24) Liu, X.; Dong, Z.-B. J. Org. Chem. 2019, 84, 11524.

(25) Rodrigues, R.; Tran, L. Q.; Darses, B.; Dauban, P.; Neuville, L. Adv. Synth. Catal. 2019, 361, 4454.

(26) Quinazolin-2-amine (3); Typical Procedure

A mixture of guanidine hydrochloride (2a; $765 \mathrm{mg}, 8 \mathrm{mmol}$ ) and $\mathrm{KOH}$ (441 mg, $8 \mathrm{mmol}$ ) was dissolved in $\mathrm{MeOH}(30 \mathrm{~mL})$ and the mixture was stirred for $10 \mathrm{~min}$ at r.t. (2-Formylphenyl)boronic acid (1; $400 \mathrm{mg}, 2.67 \mathrm{mmol}$ ) was added in one portion followed by $\mathrm{CuI}(76 \mathrm{mg}, 0.4 \mathrm{mmol})$, and the resulting mixture was heated at $70{ }^{\circ} \mathrm{C}$ overnight. The mixture was then concentrated under reduced pressure and partitioned between aq $\mathrm{NH}_{3}(30 \mathrm{~mL})$ and EtOAc $(120 \mathrm{~mL})$. The organic layer washed with brine, dried $\left(\mathrm{Na}_{2} \mathrm{SO}_{4}\right)$, and concentrated under reduced pressure. The crude product was purified by trituration with EtOAc (3 mL) to give a slightly beige solid; yield: $198 \mathrm{mg}$ (51\%); mp $194-196^{\circ} \mathrm{C}$.

${ }^{1} \mathrm{H}$ NMR $\left(400 \mathrm{MHz}\right.$, DMSO- $\left.d_{6}\right): \delta=9.10(\mathrm{~s}, 1 \mathrm{H}), 7.78(\mathrm{~d}, J=8.9$ $\mathrm{Hz}, 1 \mathrm{H}), 7.67(\mathrm{t}, J=8.5 \mathrm{~Hz}, 1 \mathrm{H}), 7.41(\mathrm{~d}, J=8.4 \mathrm{~Hz}, 1 \mathrm{H}), 7.21(\mathrm{t}$, $J=7.9 \mathrm{~Hz}, 1 \mathrm{H}), 6.82(\mathrm{~s}, 2 \mathrm{H}) .{ }^{13} \mathrm{C}$ NMR $\left(101 \mathrm{MHz}\right.$, DMSO- $\left.d_{6}\right): \delta=$ 162.4, 160.9, 151.2, 134.1, 127.9, 124.5, 122.0, 119.5. LC/MS: $\mathrm{m} / \mathrm{z}[\mathrm{M}+\mathrm{H}]^{+}$calcd for $\mathrm{C}_{8} \mathrm{H}_{8} \mathrm{~N}_{3}: 146.17$; found: 146.16 . The spectral data correspond to the reported values (see Ref. 10).

$\mathbf{N}$-Methylquinazolin-2-amine (5a)

Prepared from (2-Formylphenyl)boronic acid (1) and $N$-methylguanidine hydrochloride (4a), and purified by column chromatography [silica gel, EtOAc-PE (20 to $50 \%$ gradient)] as a yellowish solid: yield: $134 \mathrm{mg}(63 \%) ; \mathrm{mp} 81-83^{\circ} \mathrm{C} ; R_{f}=0.63$ (EtOAc). ${ }^{1} \mathrm{H}$ NMR $\left(400 \mathrm{MHz}, \mathrm{CDCl}_{3}\right): \delta=9.03(\mathrm{~s}, 1 \mathrm{H}), 7.72-7.56(\mathrm{~m}, 3 \mathrm{H})$, $7.22(\mathrm{t}, J=7.9 \mathrm{~Hz}, 1 \mathrm{H}), 5.43($ br s, $1 \mathrm{H}), 3.12(\mathrm{~d}, J=4.0 \mathrm{~Hz}, 3 \mathrm{H})$. ${ }^{13} \mathrm{C}$ NMR $\left(101 \mathrm{MHz}, \mathrm{CDCl}_{3}\right): \delta=162.01,160.50,152.44,134.37$, 127.79, 125.83, 122.72, 120.75, 28.77. HRMS: $m / z[\mathrm{M}+\mathrm{H}]^{+}$calcd for $\mathrm{C}_{9} \mathrm{H}_{10} \mathrm{~N}_{3}: 160.0875$; found: 160.0881 .

6-(Benzyloxy)- $\mathrm{N}$-methylquinazolin-2-amine (8b)

Prepared from boronic acid $\mathbf{6 b}$ and $\mathrm{N}$-methylguanidine hydrochloride (4a), and purified by column chromatography [silica gel, EtOAc-PE (20 to $60 \%$ gradient)] as a yellowish solid; yield: $150 \mathrm{mg}$ (57\%); $\mathrm{mp} 130-132{ }^{\circ} \mathrm{C}, R_{f}=0.38$ (50\% EtOAc-PE). ${ }^{1} \mathrm{H}$ NMR $\left(400 \mathrm{MHz}, \mathrm{CDCl}_{3}\right): \delta=8.88(\mathrm{~s}, 1 \mathrm{H}), 7.58(\mathrm{~d}, J=9.2 \mathrm{~Hz}, 1$ $\mathrm{H}), 7.51-7.32(\mathrm{~m}, 6 \mathrm{H}), 7.05(\mathrm{~d}, J=2.8 \mathrm{~Hz}, 1 \mathrm{H}), 5.23(\mathrm{~s}, 1 \mathrm{H}), 5.12$ (s, $2 \mathrm{H}), 3.10$ (d, $J=5.1 \mathrm{~Hz}, 3 \mathrm{H}) .{ }^{13} \mathrm{C} \operatorname{NMR}\left(101 \mathrm{MHz}, \mathrm{CDCl}_{3}\right): \delta=$ 160.67, 159.78, 154.12, 148.30, 136.70, 128.80, 128.28, 127.67, 127.23, 127.13, 120.32, 106.82, 70.55, 28.76. HRMS: $m / z$ [M + $\mathrm{H}]^{+}$calcd for $\mathrm{C}_{16} \mathrm{H}_{16} \mathrm{~N}_{3} \mathrm{O}$ : 266.1293; found: 266.1292 .

(27) Marcum, J. S.; McGarry, K. A.; Ferber, C. J.; Clark, T. B. J. Org. Chem. 2016, 81, 7963.

(28) Vantourout, J. C.; Law, R. P.; Isidro-Llobet, A.; Atkinson, S. J.; Watson, A. J. B. J. Org. Chem. 2016, 81, 3942. 